\title{
Opioid Use Disorder Treatment Decision Making And Care Navigation Upon Release From Prison: A Feasibility Study
}

This article was published in the following Dove Press journal: Substance Abuse and Rehabilitation

\author{
Caleb J Banta-Green (iD ${ }^{1,2}$ \\ Anthony S Floyd ${ }^{1,3}$ \\ Kristin Vick' \\ Jen Arthur' \\ Theresa J Hoeft ${ }^{4}$ \\ Judith I Tsui ${ }^{5}$ \\ 'Alcohol and Drug Abuse Institute, \\ University of Washington, Seattle, WA, \\ USA; '2School of Public Health, \\ Department of Health Services, \\ University of Washington, Seattle, WA, \\ USA; ${ }^{3}$ School of Public Health, \\ Department of Epidemiology, University \\ of Washington, Seattle, WA, USA; \\ ${ }^{4}$ Department of Psychiatry and \\ Behavioral Sciences, University of \\ Washington, Seattle, WA, USA; \\ ${ }^{5}$ Department of Medicine, University of \\ Washington, Seattle, WA, USA
}

Purpose: Opioid use disorder (OUD) is a medical condition that is effectively treated with medications. A major challenge in breaking the cycle of OUD and related illegal activity is seamlessly introducing medications for opioid use disorder (MOUD) as individuals leave jail or prison. We examined the feasibility of a pilot intervention to link participants to ongoing MOUD and psychosocial supports following release from custody.

Methods: The study enrolled adults with a history of OUD released from Washington State prisons to Department of Corrections (DOC) community supervision. Participants were randomized to the study intervention or comparison group. The intervention consisted of education on OUD and available treatments, support with individualized treatment decision making, and continued care navigation for 6 months to facilitate linkage to chosen treatments. Participants randomized to the control condition received referral to services in the community from their community corrections officers. A care navigation activity log documented intervention participants' intervention engagement, service utilization, and needs. Follow-up interviews were conducted at 1 and 6 months to assess satisfaction with the intervention.

Results: Fifteen participants were enrolled. All were male, most were white (86.6\%) and the average age was 36.9 years. The majority (14 of 15 participants) were near-daily heroin users with severe OUD prior to incarceration. Of the seven intervention participants, two wished to start medications immediately. Three participants reported starting buprenorphine or methadone in the subsequent follow-up period, with or without social support and/or outpatient counseling, and three reported enrolling in social support and/or outpatient counseling without medications. Participants who received the intervention reported high satisfaction. We discuss barriers and facilitators to study implementation.

Conclusion: An intervention to link participants to ongoing MOUD and psychosocial supports following release from prison had broad acceptability among participants and was feasible to implement among those recruited; however, enrollment was much lower than anticipated and the study intervention did not demonstrate the intended effect to facilitate use of MOUD immediately post-release in this small sample of participants. Given recent research showing benefits of prerelease medication initiation, the potential added benefits of this two-part intervention should be studied in systems that initiate MOUD prior to release.

Keywords: opioid use disorder, opioids, intervention development, criminal justice, treatment, feasibility study

\section{Introduction}

One-third of heroin users are incarcerated each year, primarily due to illegal activity related to their drug use. As such, there is an urgent need for interventions to facilitate
Correspondence: Caleb J Banta-Green Alcohol and Drug Abuse Institute, University of Washington, I 107 NE 45th Street, Suite 120, Box 354805, Seattle, WA 98I05-463I, USA

Email calebbg@uw.edu 
initiation of effective treatments for opioid use disorder (OUD) upon release in order to break the cycle of relapse and re-incarceration. ${ }^{1}$ The public health burden of OUD is relentless as the number of heroin and other illicit opioid users and the number having non-fatal and fatal opioid overdoses have increased significantly in recent years across the US, with pharmaceutical-type opioids serving as a common pathway into heroin and persistent overdose risk. ${ }^{2-6}$

The period immediately after release from prison is one of greatly increased risk for fatal opioid overdose. ${ }^{7,8}$ Opioid use disorder can be readily treated with agonist medications for opioid use disorder (MOUD), methadone and buprenorphine, reducing illegal activity and recidivism, improving functioning, decreasing mortality and transmission of infectious diseases like HIV and HCV, and substantially reducing costs. ${ }^{9-16}$ Long-acting naltrexone, an opioid antagonist, is a newer medication for OUD and data demonstrate effectiveness for reducing opioid use, but challenges with initiation and retention. ${ }^{17-20} \mathrm{~A}$ major challenge in breaking the cycle of OUD and illegal behaviors, and related morbidity and mortality, is seamlessly getting individuals on MOUD as they leave jail or prison. This process may be enhanced by simultaneously building interest in effective treatments among newly released inmates and facilitating access to such treatments. Given the very challenging psychosocial environments into which people are released and the high risk of relapse, initiating medications quickly is vital. 1,7,21 $^{2}$

Washington State's Opioid Response Plan identifies numerous challenges to providing effective services to people being released from prison with OUD, including: 1) identification of the population at risk, 2) inmates' often modest knowledge and misperceptions of MOUD as well as limited motivation and self-efficacy for accessing treatment, 3) lack of continuity of care between the relatively protected prison environment and effective services in the community, and 4) maintaining ongoing utilization of MOUD to support recovery and reduced consequences related to opioid use. ${ }^{22}$

Given the many gaps and barriers for people post-prison release, we developed a two-part intervention. The first part of the intervention was treatment decision making adapted from the shared decision making approach often used in health care which we adapted to OUD, moved out of the clinical setting, and had delivered by non-health care providers. ${ }^{23,24}$ In order to increase the chances of a person initiating, and due to the likelihood of needing to re-initiate care, we also provided 6 months of ongoing treatment navigation services. ${ }^{21} \mathrm{~A}$ pilot feasibility randomized controlled trial was chosen to examine the feasibility of the research procedures and the study intervention.

\section{Methods}

\section{Study Design And Setting}

The study was a pilot implementation study to assess the feasibility and acceptability of an intervention to assist persons recently released from prison to access MOUD as well as the feasibility of conducting research in this setting. The study randomly assigned participants to the intervention versus treatment-as-usual (TAU). TAU included referrals to substance use treatment and other recovery support services by community corrections. Prior to study implementation, multiple meetings took place between the study team and WA Department of Corrections (DOC) at each of the local community corrections offices (CCOs) where recruitment occurred, with all available staff to explain the intervention and the research. CCOs were in five locations in King County, Washington, south of Seattle.

\section{Eligibility Criteria}

Men or women aged 18-70 newly released from Washington State prisons to community corrections supervision in King County were recruited, ideally within 2 days of release, though they were eligible for recruitment within 30 days of release. Participants with at least mild severity criteria (at least 2 criteria in the 12 months prior to incarceration) for Diagnostic and Statistical Manual of Mental Disorders Fifth Edition (DSM-5) opioid use disorder (per the DSM-5 checklist) or a history of having been treated for opioid use disorder were eligible to participate. Other criteria included the ability to understand English and provide informed consent, and access to phone (voice or text) or email to communicate with research staff. Exclusion criteria included: under age 18 or over age 70 at time of recruitment; currently enrolled in an opioid treatment program using medications; no opioid use in the 6 months prior to incarceration; unwilling to allow access to medical or drug treatment records, as well as criminal history or criminal activity records; inability to communicate in English or provide adequate contact information to assist with follow-up; not planning on being in King County or reporting to community corrections in King County for 6 months; violent or overtly hostile/ threatening towards research staff; or expected to enter a controlled environment for 31 days or more in the next 6 months. 


\section{Study Enrollment}

Participants were recruited in local community corrections offices after prison release. Potential participants with planned releases in the coming week to community corrections were identified by DOC headquarters staff through weekly reports to local CCOs, who contacted study staff so they could be available for recruitment upon release. Potential participants were identified by DOC as having a diagnosis of OUD, or of having reported use of opioids within the 6 months prior to incarceration. When contacted, the two female care navigators went on-site during the expected days of release, typically within 2 days of release, to recruit and enroll the potential participant during their first visit at the community corrections office. The two patient navigators had professional experience with substance use disorders and the criminal justice system. The care navigators attempted to meet potential participants during the initial visit, however this was not always logistically possible. Potential participants received a flyer introducing the study in their community corrections intake materials provided by DOC at CCOs. Community corrections officers did not attempt to recruit participants directly, but asked whether participants were interested in meeting with study staff. In the event that a released person did not appear at that facility or needed more time for study procedures, study staff returned on subsequent days. Care navigators could recruit participants within 30 days of release, but aimed to do so within a week.

Written informed consent was obtained from all individuals who wished to participate in the study. For potential participants who did not want to participate, navigators made observations, not linked to a study identifier, about demographics (apparent race, gender, age range) and reasons why they did not want to participate. This information helped identify whether those who enrolled in the study were representative of the total eligible population. Participants were not compensated for any study procedures per DOC policy. The University of Washington Human Subjects Division reviewed and approved all study procedures.

\section{Screening And Randomization}

Once consent was obtained, participants were screened for eligibility criteria (above). They were then asked demographic information (race, ethnicity, gender, age), whether they were willing to share medical and criminal history, and their use of opioids in the period prior to incarceration. Participants also completed the DSM-5 checklist for opioid use disorder to document severity of use disorder. Participants were required to be classified as having at least mild severity based on their pre-incarceration use of opioids. Participants were randomized to receive either usual care or the study intervention. We attempted to recruit equal numbers of participants in the intervention and comparison arms of the study. Randomization was implemented in the REDCap data capture system.

\section{Baseline Assessments}

Assessments included: opioid use and treatment history (opioid use, overdose history, past treatment and feelings about future treatment); education, employment, income and housing; overdose risk factors; World Health Organization Alcohol, Smoking and Substance Involvement Screening Test (WHO-ASSIST v.3); and the EQ-5D-3L, a validated quality of life measure.

\section{Follow-Up Visits}

Participants were followed for 6 months with phone interviews at 1 and 6 months after enrollment conducted by a research support center at the University of Washington. Assessments given at the baseline interview were repeated at each follow-up point. In addition to the assessments, participants were asked questions in the intervention arm about their satisfaction with the treatment navigation process.

\section{Intervention Components}

The intervention included an initial education/decisionfacilitation session with the care navigator based upon a patient-centered discussion of their opioid use and options for treatment. The intervention aimed to build motivation and self-efficacy for and ready access to adequate coordinated care for individuals recently released from prison with OUD. The goal was for participants to make fully informed and self-directed/autonomous decisions regarding OUD treatment which included: recovery support groups, individual or group addiction counseling, and/or MOUD. Treatment services were selected by the study participants and provided by community-based programs and providers not associated with the study.

The education/decision-facilitation intervention drew from prior development of patient-centered tools for opioid users. ${ }^{25-28}$ Research has shown that many individuals with OUD have an incomplete or inaccurate understanding of their condition and carry misperceptions of the treatment medications and the modes of care delivery. ${ }^{29}$ Therefore, the intervention was designed to provide accurate information and guide patients to make an informed decision consistent with their personal preferences. 
Additionally, understanding participant experiences, preferences, and the etiology of their perspectives informs future interventions in this population. The intervention utilized materials available from the Substance Abuse and Mental Health Services Administration (SAMHSA) regarding current MOUD (i.e. methadone, buprenorphine and extended-release naltrexone) and incorporated this information into a guide that also describes locally available treatment resources. ${ }^{30}$

The trained interventionist/treatment navigator reviewed all OUD treatment options including MOUD, helped participants decide which (if any) was best for them, and connected them to their treatment(s) of choice. This involved helping them schedule an initial appointment for MOUD, addiction counseling, and/or locating an accessible recovery support group. Treatment options were summarized with the participant documenting the pros and cons of each option based upon their preferences and situation (See Appendix). If a treatment choice was made during the baseline visit, we used a customized online resource map (See Appendix) to help locate desired services taking into consideration where they were living and transportation options.

The second part of the intervention was ongoing patient OUD treatment navigation to facilitate initiation and maintenance of treatment services for 6 months following study enrollment. The patient navigator explained to participants:

After today, I will be available to work with you for six months to talk through how things are going, if you'd like to try a different type of treatment, and to help you get back on treatment if you stop. Treating opiate use disorder is like treating other health conditions. Often different methods need to be tried until the one that works best is found. I will stick with you through this process, even if you relapse.

Navigators attempted to contact participants at least weekly by phone, text, e-mail or in-person based on participant preference to determine if they needed assistance, or if their treatment needs had changed. Elements of treatment decision making could be repeated as necessary as preferences and situations evolved. If participants wanted to opt into a treatment option after previously declining, the navigator identified locations that were convenient and made contact with treatment providers to help facilitate the process where possible. The intervention adapted previous work implementing patient navigation services for inmates upon release designed to increase access to healthcare. ${ }^{21}$
Due to the high risk of fatal opioid overdose following prison release, participants in both the comparison and intervention groups were offered opioid overdose education and take-home-naloxone for opioid overdose reversal. Providing take-home-naloxone to high-risk individuals is supported by the research literature as well as recent policy guidance by the Centers for Disease Control. ${ }^{31-33}$ Participants in the comparison group received standard referral to services in the community from their community corrections officers. Referrals included a variety of social services including OUD treatment options.

Navigators received several days of training on study procedures, informed consent process, electronic data collection tools, and basic motivational interviewing skills covered in an 8-hr training. Training was provided on basic use and scoring of the DSM-5 checklist.

\section{Results}

\section{Study Enrollment}

DOC data management staff identified 125 individuals being released to community corrections during the study period. Of those 125, 63 were approached for participation at community corrections offices, 44 individuals declined to participate or were ineligible. The remaining 19 individuals were further screened for eligibility and 15 were found eligible, randomized, and enrolled in the study. Seven participants were randomized to receive the study intervention. See Figure 1 for a CONSORT diagram.

Individuals who did not participate mentioned multiple reasons for not doing so. Most of the individuals who did not participate did not because they were "not currently using opioids" (56.8\%) many also expressed a lack of time to participate due to many DOC requirements and life challenges. More than a quarter also noted that they either never used opioids (27.3\%), or primarily used substances other than opioids (29.5\%). The main type of substance other than opioids used was stimulants.

\section{Baseline Demographics And Substance Use-Related History}

Table 1 describes demographic characteristics of enrolled participants, those screened and not enrolled, and those who were not formally screened. Across all groups, the majority were middleaged males. While the 15 enrolled participants were majority white, the 44 individuals who were not screened were more diverse with 34\% African-Americans. Most enrolled participants (14 of 15) had a history of heroin use and among these 


\section{Treatment Navigation CONSORT Flow Diagram}

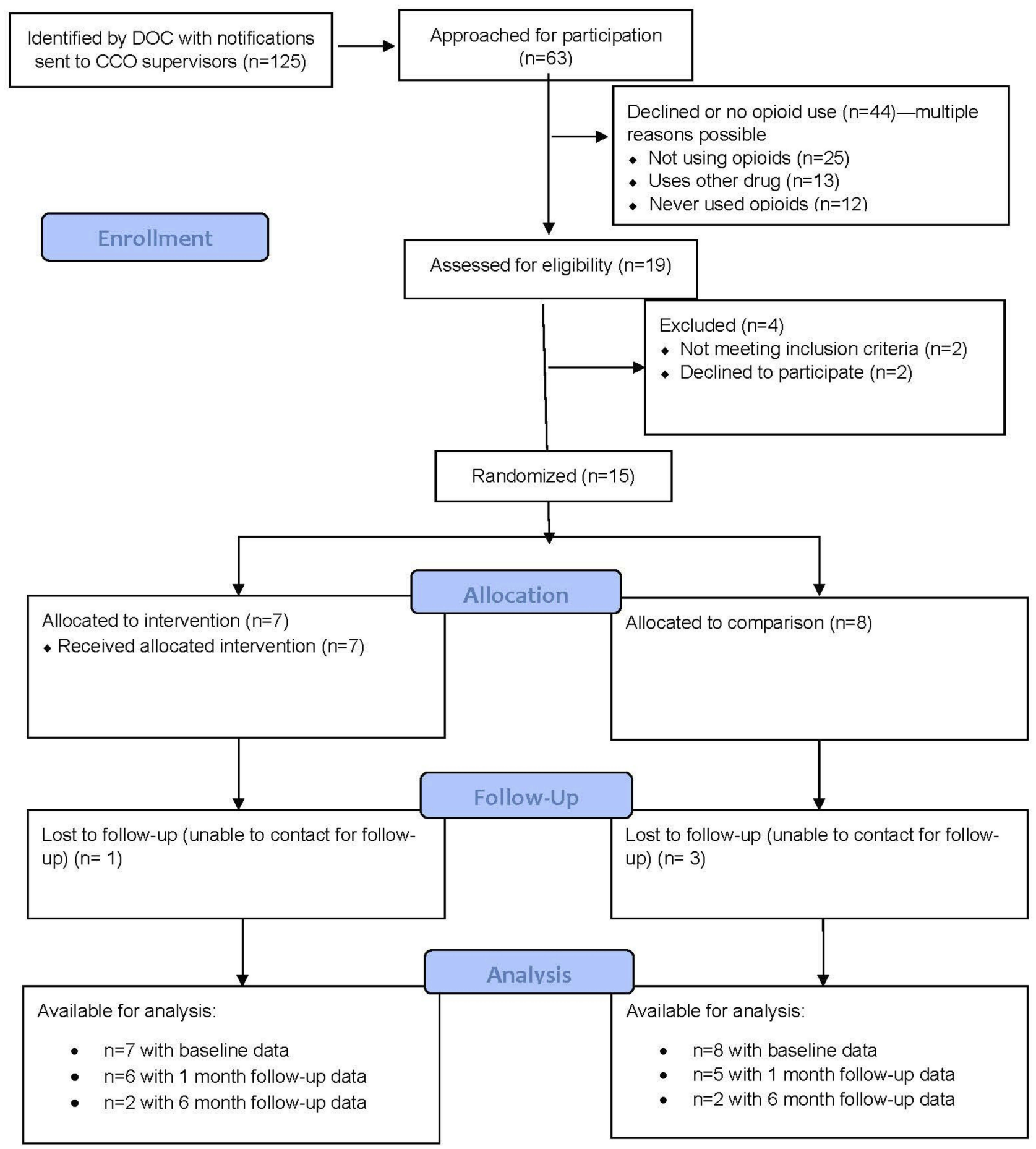

Figure I CONSORT flow diagram.

14 participants, the mean number of days of use in the 30 days prior to incarceration was 27.8. Slightly more than half, eight participants, used prescription-type opioids with a mean of 16.8 days of use in the 30 days prior to incarceration. Enrolled participants all had very high severity of OUD per DSM-5 ( $>9$ of 11 score). Ten of the 15 enrolled participants had used MOUD previously, including 9 who had been on methadone from an opioid treatment program for OUD, 6 who had been prescribed buprenorphine for OUD, and one who had been prescribed naltrexone for OUD. Two-thirds of participants 
Table I Characteristics Of People Screened For Eligibility And Enrolled

\begin{tabular}{|c|c|c|c|c|c|}
\hline & $\begin{array}{l}\text { Intervention } \\
\text { Group } n=7\end{array}$ & $\begin{array}{l}\text { Comparison } \\
\text { Group } n=8\end{array}$ & $\begin{array}{l}\text { Total Screened } \\
\text { And Enrolled } n=15\end{array}$ & $\begin{array}{l}\text { Screened, Not } \\
\text { Enrolled } n=4\end{array}$ & $\begin{array}{l}\text { Not Screened*(Observed } \\
\text { Characteristics) } n=44\end{array}$ \\
\hline \multirow[t]{2}{*}{ Age mean (SD) } & $36.6(7.3)$ & $37.1(9.6)$ & $36.9(8.3)$ & $35.3(10.5)$ & \\
\hline & & & & & n (\%) \\
\hline $\begin{array}{l}\text { Observed age range } \\
\text { 20s: } \\
\text { 30s: } \\
\text { 40s: } \\
\text { 50s: } \\
\text { 60s: } \\
\text { Do not know: }\end{array}$ & & & & & $\begin{array}{l}3(7) \\
17(39) \\
10(23) \\
4(9) \\
1(2) \\
9(21)\end{array}$ \\
\hline Male & $7(100)$ & $8(100)$ & $15(100)$ & $4(100)$ & $37(86.0)$ \\
\hline \multicolumn{6}{|l|}{$\begin{array}{l}\text { Observed race/ethnicity } \\
\text { (multiple could be selected) }\end{array}$} \\
\hline $\begin{array}{l}\text { Hispanic } \\
\text { White } \\
\text { Black } \\
\text { American Indian/Alaska } \\
\text { Native } \\
\text { Race Other } \\
\text { Do not know }\end{array}$ & $\begin{array}{l}0(0) \\
7(100) \\
0(0) \\
0(0) \\
0(0)\end{array}$ & $\begin{array}{l}0(0) \\
6(75) \\
\text { I }(12.5) \\
0(0) \\
I(12.5)\end{array}$ & $\begin{array}{l}0(0) \\
\text { I3 (87) } \\
\text { I (7) } \\
0(0) \\
\text { I (7) }\end{array}$ & $\begin{array}{l}\text { I }(25) \\
\text { I (25) } \\
\text { I (25) } \\
\text { I (25) } \\
\text { I (25) }\end{array}$ & $\begin{array}{l}19(43) \\
15(34) \\
1(2) \\
9(22)\end{array}$ \\
\hline $\begin{array}{l}\text { Mean days used heroin in last } \\
30^{* *} \text { (SD) }\end{array}$ & $\begin{array}{l}24.8(10.4) \\
n=6\end{array}$ & $30.0(0) n=8$ & $27.8(6.9) n=14$ & $30(0) n=3$ & \\
\hline $\begin{array}{l}\text { Mean days used Rx opioids in } \\
\text { last } 30^{* *}(\mathrm{SD})\end{array}$ & $\begin{array}{l}19.8(13.9) \\
n=5\end{array}$ & $\begin{array}{l}11.7(15.9) \\
n=3\end{array}$ & $16.8(14.2)=8$ & $30(0) n=1$ & \\
\hline $\begin{array}{l}\text { Mean DSM-5 OUD Checklist } \\
\text { Severity (SD) [Range } 0-1 \mathrm{I}]\end{array}$ & $10.9(0.4) n=7$ & $10.9(0.4) n=8$ & $10.9(0.4) n=15$ & $10.5(0.7) n=2$ & \\
\hline
\end{tabular}

Notes: *Declined or no opioid use. **Prior to incarceration.

$(n=10)$ had experienced an opioid overdose, and all but one had witnessed an opioid overdose. Importantly, participants felt their overdose risk was low, with most participants $(80 \%)$ responding that it was extremely unlikely that they would experience an opioid overdose in the next year, and $66.7 \%$ responding that they were not concerned about experiencing an opioid overdose. At baseline, all of the intervention participants accepted takehome naloxone, while only half of the comparison group did so (all were offered)

\section{Study Outcomes}

Because of the small final sample size, we were unable to make formal comparisons between participants in the comparison arm $(n=8)$ and the intervention arm $(n=7)$. The remaining sections focus on describing the intervention group. We were able to locate 6 of 7 intervention participants for 1-month follow-up, and two at 6 months.

\section{Treatment Navigation Utilization, Themes And Satisfaction}

On average, navigators initiated contact with participants 4 times more (20 attempts on average) than participants initiated contact with navigators ( 5 attempts on average) during the treatment navigation period. As a feasibility study, it was not pre-specified what the target goals were for participant communication. The goal was to provide a constant presence, so navigators would regularly continue to reach out to participants, initially weekly, even if the lines of communication appeared to be broken. The most common issue voiced by participants with the navigators was drug craving (78\%). Other concerns included mental health $(57 \%)$, employment $(57 \%)$, enrolling in counseling (57\%), initiating MOUD (43\%), and housing (43\%).

Participant satisfaction with the intervention, collected at 1 month $(n=6)$ and 6 months $(n=2)$ was very positive across all 
categories, with participants indicating they were "satisfied" or "very satisfied" in terms of being: treated respectfully; provided with accurate information; provided with decision making support; and helped with accessing treatment and other services. Participants also cited the ongoing contact and support they received from the navigators as contributing to the overall high appraisal of the intervention.

\section{Examples Of Participants' Initial And Ongoing Treatment Interests}

Here we provide sample case summaries of three intervention participants, describing their treatment histories, initial treatment choices, and eventual treatment utilization taken from notes during the navigation period following treatment decision making.

Case A: White male, age range 30-39. Treatment history included recovery support groups, inpatient treatment, outpatient counseling, and OUD treatment with methadone and buprenorphine. He had also used buprenorphine without a prescription. He was a daily methadone (from an opioid treatment program) and cannabis user and occasional sedative user in the 3 months before incarceration (heavy heroin use prior to methadone treatment). Initial treatment preference when the intervention was provided was recovery support groups and outpatient counseling (which may have been DOC mandated). Early in treatment navigation, this participant reported that he thought medications (especially opioid agonists) should be reserved for those actively in addiction. He disliked the first outpatient treatment agency he went to and rescheduled the second location several times before completing an assessment. The participant began having cravings and used non-opioid drugs to curb them. Eventually, he wanted to be connected with a buprenorphine prescriber as well as outpatient addiction counseling, so he was connected with a local clinic that did both. After getting into treatment, the participant did very well, obtaining a fulltime employment, private housing, and avoiding relapse.

Case B: White male, age range 30-39. Treatment history included recovery support groups, inpatient treatment, and OUD treatment with methadone. Participant was a daily heroin, prescription opioid, and cannabis user in the 3 months before incarceration. Treatment preference at the outset was outpatient counseling and buprenorphine or naltrexone, so he was connected with a provider who prescribed both medications so they could decide together. The participant missed and rescheduled his medical intake appointment a few times, but eventually made it and was inducted on buprenorphine. The participant initially did not like how he felt on the medication, noting he felt too much opioid effect, so his dose was lowered which seemed to improve how he felt on it, but did not resolve his discomfort completely. After more discussion with his provider, he stayed on buprenorphine and later described that things were going well with treatment. After that the navigator lost contact with the participant for several months until he reinitiated contact looking for help finding methadone treatment. The navigator connected the participant with a methadone treatment provider, but then again lost contact. It is unknown whether the participant began treatment with methadone.

Case C: White male, age range 40-49. Treatment history included recovery support groups, inpatient treatment, outpatient counseling, and OUD treatment with methadone and buprenorphine. Participant was a daily prescription-type opioid, methamphetamine, and alcohol user and occasional heroin user in the 3 months before incarceration. His initial treatment preferences were recovery support groups, outpatient addiction counseling, and naltrexone. He had favorable views of buprenorphine but did not want to go on an opioid medication unless he was actively addicted. Attempts to connect him with a provider were unsuccessful due to an inflexible work schedule and unreliable communication. Contact with the participant was lost until he responded to a call, saying he relapsed and was interested in buprenorphine. He was connected with a provider, but soon after contact was lost again. We discovered that the participant had an arrest warrant and was arrested during the study period.

Other Cases: Among the four other intervention participants all reported prior treatment with MOUD and all indicated a preference for recovery support groups and outpatient addiction counseling at the time of the intervention. The three that were interviewed at 1-month follow-up indicated that their baseline treatment preferences had not changed: non-MOUD, recovery support groups, and outpatient counseling. Two of these participants were arrested during the 6-month study period.

\section{Discussion}

Enrolled participants had broad exposure to many kinds of treatment options in the past with the majority having been on methadone and/or buprenorphine. However, while most were interested in recovery support groups or addiction counseling initially, few were interested initially in MOUD, perhaps seeing their significant time off of opioids and their lack of physical dependence as having ameliorated their opioid use disorder, a very common misunderstanding of OUD. ${ }^{34}$ In fact, research shows that 
approximately three-quarters of people with OUD relapse within a few months of release..$^{35,36}$

For several intervention participants care navigation began to pay dividends after several months as the established relationship and communication seemed to allow participants to express concerns around craving. This was often manifested as a brief regular check in by the care navigator which resulted in a response from the participant that they had concerns or were not doing well. The navigator would then quickly establish contact, have a conversation, and work to implement a plan to access services, in these instances initiating buprenorphine. This timing of a lag to medication initiation is understandable in the context of a release from prison and suggests that care navigation lasting multiple months, even if the contacts are initially infrequent and may appear only modestly substantive, may be worthwhile as they provide a relational and communication dynamic which can be responsive when it is, eventually, needed.

In addition to their low perceived risk of relapse, participants also had low overdose risk perception - all of the intervention participants at baseline responded that it was extremely unlikely for them to experience opioid overdose. This is contrary to reality as documented by research demonstrating very high overdose risk postincarceration. ${ }^{7}$ The overdose education component of the intervention, combined with the ongoing attention of the navigators may have been helpful for some participants once it became apparent to them that they were vulnerable to craving or resumed opioid use.

The intervention may also have been beneficial for enhancing understanding of OUD and care options including all three FDA approved medications. Some participants were familiar with buprenorphine but did not have a good understanding of where to initiate treatment. Some only had experience with using un-prescribed buprenorphine outside of a medical setting, as reported elsewhere and found to be associated with future successful prescribed buprenorphine use. ${ }^{37}$ A few participants had heard of naltrexone, but again did not have a good understanding of how it worked or where to initiate treatment. Some confused naltrexone with naloxone. While naltrexone does not have the same level of proven effectiveness of methadone or buprenorphine, it is still a medication that some participants are interested in as some did not wish to become dependent on an opioid again and may be of value to some. ${ }^{18,20}$ Re-initiating opioids, and in turn becoming physically dependent, is a concern identified in previous research, although interest in these medications and initiation is common as well. ${ }^{1}$
Navigators also noted that some participants had difficulty with making decisions, perhaps as a result of their very controlled environment while incarcerated, or the number of tasks required to be completed upon release (e.g. reporting to CCOs, obtaining employment, finding housing). Future interventions might consider modifying the treatment decision making approach to ask participants if they would like a more directive intervention, perhaps asking them if they would like some examples of what has been successful for other people in similar situations with similar preferences. While the intervention included language explicitly describing relapse and overdose rates for people releasing from incarceration and the mortality benefits of methadone and buprenorphine (See Appendix), it did not provide direct advice to utilize medications pre-, or immediately post-, release. Future intervention development and research should consider the appropriateness of care navigators providing such direct advice given the proven benefit of opioid agonist medications for the treatment of opioid use disorder ${ }^{20,38}$ and research indicating the benefit of prescriber advice to use medications for alcohol use disorder. ${ }^{39}$

Despite strong buy-in and support from DOC at the local and state levels to conduct this research, there were significant challenges to the feasibility of implementing this clinical trial in community corrections settings. This resulted in much lower than expected recruitment. The small number of participants enrolled in the study led to an inability to create statistical comparisons across groups regarding outcomes of interest, rather findings presented were qualitative in nature and based upon care navigation notes. No women were enrolled despite proximity to a women's prison in the region and approximately $15 \%$ of potential participants being female, this despite the navigators being female which could have increased chances of engagement. The population enrolled was much less likely to be African American than those identified as potentially eligible by DOC and the group screened for the study. The reasons for this disparity are unknown but could include: lower rates of presenting to the community corrections offices, lower referral rates by corrections staff, African American participants may not have perceived a fit with white female care navigators, or differential interest in the intervention on the part of potential participants. This under-representation of African Americans in enrollment is very problematic given the relatively high proportion of potentially eligible participants who were African American and the disproportionately high rate of African Americans in prison in Washington State. Also of concern is the under-representation of women, in particular given that an all-female prison is located in the region. Future intervention 
development and research should explicitly examine and attempt to ameliorate these issues.

Among the recruitment challenges was apparent misclassification of potential participants identified as being potentially eligible with opioid use disorder or past opioid use. Many of the misclassified potential participants had a history of other substance use, not related to opioids. Because self-reports of opioid use were used both by DOC records review as an automated prescreen to identify potentially eligible people and during the initial approach by study staff, we cannot say with certainty whether our search criteria were poorly specified, or whether potential participants were not forthcoming about past opioid use. We were also unable to provide any form of participant compensation, which may have decreased our recruitment rate. This lack of compensation was due to a long-standing DOC policy precluding compensation, which the researchers formally appealed and were denied.

Some logistical issues in recruitment were inmate release schedules that were changed with little notice, and inmates who were released presenting to CCOs with no time available for enrollment, requiring subsequent visits that were difficult to schedule. Community corrections officers were also very busy, working in a busy workplace setting and did not always have the time to initiate contact with study staff when a potential participant was available. Previous research on incorporating methadone and buprenorphine into corrections has identified many similar barriers. ${ }^{16,40}$ Research shows the feasibility and positive outcomes associated with starting MOUD prior to release, and given the challenges we identified post-release, suggests this may be a more successful approach. ${ }^{41,42}$ In 2019 DOC began buprenorphine inductions prior to release, at the time of this study no MOUD were available for those in prison.

\section{Conclusion}

Treatment decision making and navigation from a care navigator were well received among participants, but was not feasible subsequent to prison release in the community corrections setting due to the logistical issues with the corrections systems and competing demands on people being released from prison. The OUD and MOUD educational components appear to be a good way to provide evidence-based information to participants. The treatment decision making process may enhance understanding of OUD and MOUD and ongoing navigation has the potential to be helpful in keeping participants engaged. Given recent research showing benefits of prerelease medication initiation, the potential added benefits of this two-part intervention should be studied in systems that initiate MOUD prior to release.

\section{Acknowledgments}

This study was supported by funds from Arnold Ventures in 2017-2019. The views expressed in this report are the authors' and do not necessarily reflect the views of the funder. Study data were collected and managed using REDCap electronic data capture tools hosted at the Institute of Translational Health Sciences. REDCap at ITHS is supported by the National Center for Advancing Translational Sciences of the National Institutes of Health under Award Number UL1 TR002319. Thank you to Barbara Burke, Kimberli Dewing, Vasiliki Georgoulas-Sherry, Steven Hammond, Paige Harrison, Dawn Williams, Ingrid Binswanger, Shane Mueller, and administrative support staff at the University of Washington, Alcohol and Drug Abuse Institute.

\section{Disclosure}

Caleb J Banta-Green reports grants from Laura and John Arnold Foundation, during the conduct of the study. Kristin Vick is currently affiliated with the Study and Advancement of Justice Effectiveness Center at the University of Washington in Seattle, WA. Jen Arthur is currently affiliated with Country Doctor Community Health Centers in Seattle, WA. The authors report no other conflicts of interest in this work.

\section{References}

1. Fox AD, Maradiaga J, Weiss L, Sanchez J, Starrels JL, Cunningham CO. Release from incarceration, relapse to opioid use and the potential for buprenorphine maintenance treatment: a qualitative study of the perceptions of former inmates with opioid use disorder. Addict Sci Clin Pract. 2015;10:2. doi:10.1186/s13722-014-0023-0

2. Rudd RA, Aleshire N, Zibbell JE, Gladden M Increases in Drug and Opioid Overdose Deaths - United States, 2000-2014. Available from: http:// www.cdc.gov $/ \mathrm{mmwr} /$ preview $/ \mathrm{mmwrhtml} / \mathrm{mm} 6450 \mathrm{a} 3 . h \mathrm{tm}$ ?s_cid= mm6450a3 w. Published 2016. Accessed October 11, 2019.

3. Vivolo-Kantor AM, Seth P, Gladden RM, et al. Vital signs: trends in emergency department visits for suspected opioid overdoses - United States, July 2016-September 2017. MMWR Morb Mortal Wkly Rep. 2018;67(9):279-285. doi:10.15585/mmwr.mm6709e1

4. O'Donnell JK, Halpin J, Mattson CL, Goldberger BA, Gladden RM. Deaths involving fentanyl, fentanyl analogs, and U-47700 - 10 States, July-December 2016. MMWR Morb Mortal Wkly Rep. 2017;66(43):1197-1202. doi:10.15585/mmwr.mm6643e1

5. Jones CM, Logan J, Gladden RM, Bohm MK. Vital signs: demographic and substance use trends among heroin users - United States, 2002-2013. MMWR Morb Mortal Wkly Rep. 2015;64(26):719-725.

6. Peavy KM, Banta-Green CJ, Kingston S, Hanrahan M, Merrill JO, Coffin PO. "Hooked on" prescription-type opiates prior to using heroin: results from a survey of syringe exchange clients. J Psychoactive Drugs. 2012;44 (3). doi:10.1080/02791072.2012.704591

7. Binswanger IA, Blatchford PJ, Mueller SR, Stern MF. Mortality after prison release: opioid overdose and other causes of death, risk factors, and time trends from 1999 to 2009. Ann Intern Med. 2013;159(9):592600. doi:10.7326/0003-4819-159-9-201311050-00005

8. Merrall ELC, Kariminia A, Binswanger IA, et al. Meta-analysis of drug-related deaths soon after release from prison. Addiction. 2010;105(9):1545-1554. doi:10.1111/j.1360-0443.2010.02990.x 
9. Clark RE, Samnaliev M, Baxter JD, Leung GY. The evidence doesn't justify steps by state medicaid programs to restrict opioid addiction treatment with buprenorphine. Health Aff. 2011;30(8):1425-1433. doi: $10.1377 /$ hlthaff.2010.0532

10. Tkacz J, Volpicelli J, Un H, Ruetsch C. Relationship between buprenorphine adherence and health service utilization and costs among opioid dependent patients. $J$ Subst Abuse Treat. 2014;46(4):456-462. doi:10.1016/j.jsat.2013.10.014

11. Nordlund D, Estee S, Felver S. Methadone and Non-Methadone Treatment of Persons Addicted to Opiates Results in Lower Health Care Costs and Reduced Arrests and Convictions. Olympia, WA: Washington State Department of Social and Health Services; 2004.

12. Tsui JI, Evans JL, Lum PJ, Hahn JA, Page K. Association of opioid agonist therapy with lower incidence of hepatitis $\mathrm{C}$ virus infection in young adult injection drug users. JAMA Intern Med. 2014;174 (12):1974-1981. doi:10.1001/jamainternmed.2014.5416

13. Nolan S, Dias Lima V, Fairbairn N, et al. The impact of methadone maintenance therapy on hepatitis $\mathrm{C}$ incidence among illicit drug users. Addiction. 2014;109(12):2053-2059. doi:10.1111/add.12682

14. White B, Dore GJ, Lloyd AR, Rawlinson WD, Maher L. Opioid substitution therapy protects against hepatitis $\mathrm{C}$ virus acquisition in people who inject drugs: the HITS-c study. Med J Aust. 2014;201 (6):326-329. doi:10.5694/mja13.00153

15. MacArthur GJ, Minozzi S, Martin N, et al. Opiate substitution treatment and HIV transmission in people who inject drugs: systematic review and meta-analysis. $B M J$. 2012;345:e5945. doi:10.1136/bmj. e5945

16. Kinlock TW, Gordon MS, Schwartz RP, Fitzgerald TT, O'Grady KE. A randomized clinical trial of methadone maintenance for prisoners: results at 12 months postrelease. J Subst Abuse Treat. 2009;37 (3):277-285. doi:10.1016/j.jsat.2009.03.002

17. Lee JD, Nunes EV, Novo P, et al. Comparative effectiveness of extended-release naltrexone versus buprenorphine-naloxone for opioid relapse prevention (X:BOT): a multicentre, open-label, randomised controlled trial. Lancet. 2018;391(10118):309-318. doi:10.1016/S0140-6736(17)32812-X

18. Lee JD, Friedmann PD, Kinlock TW, et al. Extended-release naltrexone to prevent opioid relapse in criminal justice offenders. $N$ Engl $J$ Med. 2016;374(13):1232-1242. doi:10.1056/NEJMoa1505409

19. Lee JD, McDonald R, Grossman E, et al. Opioid treatment at release from jail using extended-release naltrexone: a pilot proof-of-concept randomized effectiveness trial. Addiction. 2015;110(6):1008-1014. doi:10.1111/add.12894

20. Larochelle MR, Bernson D, Land T, et al. Medication for opioid use disorder after nonfatal opioid overdose and association with mortality. Ann Intern Med. 2018. doi:10.7326/M17-3107

21. Binswanger IA, Whitley E, Haffey P-R, Mueller SR, Min S-J. A patient navigation intervention for drug-involved former prison inmates. Subst Abus. 2015;36(1):34-41. doi:10.1080/ 08897077.2014.932320

22. Workgroup WSOR. WA State Opioid Response Plan. Olympia. 2018. Available from: https://www.doh.wa.gov/Portals/1/Documents/1000/ 140-182-StateOpioidResponsePlan.pdf. Accessed October 11, 2019.

23. Elwyn G, Frosch D, Thomson R, et al. Shared decision making: a model for clinical practice. J Gen Intern Med. 2012;27(10):13611367. doi:10.1007/s11606-012-2077-6

24. Joosten EAG, de Jong CAJ, de Weert-van Oene GH, Sensky T, van der Staak CPF. Shared decision-making reduces drug use and psychiatric severity in substance-dependent patients. Psychother Psychosom. 2009;78(4):245-253. doi:10.1159/000219524

25. Banta-Green CJ, Von Korff M, Sullivan MD, Merrill JO, Doyle SR, Saunders K. The prescribed opioids difficulties scale: a patient-centered assessment of problems and concerns. Clin J Pain. 2010;26:6. doi:10.1097/AJP.0b013e3181e103d9
26. Banta-Green CJ, Coffin PO, Merrill JO, et al. Impacts of an opioid overdose prevention intervention delivered subsequent to acute care. Inj Prev. 2018. injuryprev-2017-042676. doi:10.1136/injuryprev2017-042676

27. Kelly SM, Brown BS, Katz EC, et al. A comparison of attitudes toward opioid agonist treatment among short-term buprenorphine patients. Am J Drug Alcohol Abuse. 2012;38(3):233-238. doi:10.3109/00952990.2011.643983

28. Schwartz RP, Kelly SM, O'Grady KE, et al. Attitudes toward buprenorphine and methadone among opioid-dependent individuals. $\mathrm{Am} \mathrm{J}$ Addict. 2008;17(5):396-401. doi:10.1080/10550490802268835

29. Sindicich N, Zador D, Larney S, Dolan K. Patient Motivations, Perceptions and Experiences of Opioid Substitution Therapy in Prison. Technical Report Number 332 Funded by Justice Health and Forensic Mental Health Network. Sydney, Australia: National Drug and Alcohol Research Centre; 2016.

30. Substance Abuse and Mental Health Services Administration. SAMHSA Opioid Overdose Prevention Toolkit.; 2016. Available from: https://bjatta.bja.ojp.gov/sites/default/files/naloxone/SMA164742.pdf. Accessed May 7, 2018.

31. Coffin PO, Sullivan SD. Cost-effectiveness of distributing naloxone to heroin users for lay overdose reversal. Ann Intern Med. 2013;158 (1):1-9. doi:10.7326/0003-4819-158-1-201301010-00003

32. Walley AY, Xuan Z, Hackman HH, et al. Opioid overdose rates and implementation of overdose education and nasal naloxone distribution in Massachusetts: interrupted time series analysis. BMJ. 2013;346:f174. doi:10.1136/bmj.f174

33. Dowell D, Haegerich TM, Chou R. CDC guideline for prescribing opioids for chronic pain-United States, 2016. JAMA. 2016. doi:10.1001/jama.2016.1464

34. O'Brien CP, McLellan AT. Myths about the treatment of addiction. Lancet. 1996. doi:10.1016/S0140-6736(96)90409-2

35. Cepeda JA, Niccolai LM, Lyubimova A, Kershaw T, Levina O, Heimer R. High-risk behaviors after release from incarceration among people who inject drugs in St. Petersburg, Russia. Drug Alcohol Depend. 2015;147:196-202. doi:10.1016/j. drugalcdep.2014.11.021

36. Binswanger IA, Nowels C, Corsi KF, et al. Return to drug use and overdose after release from prison: a qualitative study of risk and protective factors. Addict Sci Clin Pract. 2012;7(1):3. doi:10.1186/ 1940-0640-7-3

37. Fox AD, Chamberlain A, Sohler NL, Frost T, Cunningham CO. Illicit buprenorphine use, interest in and access to buprenorphine treatment among syringe exchange participants. J Subst Abuse Treat. 2015;48 (1):112-116. doi:10.1016/j.jsat.2014.07.015

38. Sordo L, Barrio G, Bravo MJ, et al. Mortality risk during and after opioid substitution treatment: systematic review and meta-analysis of cohort studies. BMJ. 2017;357:j1550. doi:10.1136/bmj.j1550

39. Anton RF, O'Malley SS, Ciraulo DA, et al. Combined pharmacotherapies and behavioral interventions for alcohol dependence. JAMA. 2006;295(17):2003. doi:10.1001/jama.295.17.2003

40. Kinlock TW, Gordon MS, Schwartz RP, Fitzgerald TT. Developing and implementing a new prison-based buprenorphine treatment program. $J$ Offender Rehabil. 2010;49(2):91-109. doi:10.1080/ 10509670903534951

41. Magura S, Lee JD, Hershberger J, et al. Buprenorphine and methadone maintenance in jail and post-release: a randomized clinical trial. Drug Alcohol Depend. 2009;99(1-3):222-230. doi:10.1016/j. drugalcdep.2008.08.006

42. Green TC, Clarke J, Brinkley-Rubinstein L, et al. Postincarceration fatal overdoses after implementing medications for addiction treatment in a statewide correctional system. JAMA Psychiatry. 2018;75 (4):405. doi:10.1001/jamapsychiatry.2017.4614 


\section{Publish your work in this journal}

Substance Abuse and Rehabilitation is an international, peerreviewed, open access journal publishing original research, case reports, editorials, reviews and commentaries on all areas of addiction and substance abuse and options for treatment and rehabilitation. The manuscript management system is completely online and includes a very quick and fair peer-review system. Visit http://www.dovepress. com/testimonials.php to read real quotes from published authors.

Submit your manuscript here: http://www.dovepress.com/substance-abuse-and-rehabilitation-journal 